\title{
Practical determination of individual element resistive states in selectorless RRAM arrays
}

\author{
Alexander Serb Member, IEEE, William Redman-White Fellow, IEEE, Christos Papavassiliou Senior \\ Member, IEEE,, Themistoklis Prodromakis Senior Member, IEEE,
}

\begin{abstract}
Three distinct methods of reading multi-level crosspoint resistive states from selector-less RRAM arrays are implemented in a physical system and compared for read-out accuracy. They are: the standard, direct measurement method and two methods that attempt to enhance accuracy by computing cross-point resistance on the basis of multiple measurements. Results indicate that the standard method performs as well as or better than its competitors. SPICE simulations are then performed with controlled amounts of non-idealities introduced in the system in order to test whether any technique offers particular resilience against typical practical imperfections such as crossbar line resistance. We conclude that even though certain non-idealities are shown to be minimised by different circuit-level read-out strategies, line resistance within the crossbar remains an outstanding challenge.
\end{abstract}

Index Terms - Crossbar, RRAM, SPICE, measurement technique, analogue circuits, memory, device characterisation

\section{INTRODUCTION}

Resistive Random-Access Memory (RRAM) is a promising, emerging, beyond-Moore memory technology whereby storage nodes operate on the basis of the resistive switching phenomenon. RRAM systems exhibit small size and good scalability (down to $8 \times 8 \mathrm{~nm}$ node size reported in the literature) [1], [2], multi-state memory storage [3], low-power operation [4] and rely on the use of simple, 2-terminal devices; all highly desirable characteristics for applications ranging from large, industrial memory cells to neuromorphic applications. In neuromorphic engineering RRAM is seen as a possible means of linking pre- and post-synaptic neurons through areaeffective artificial synapses [5]; currently a fundamental stumbling block towards the development of large-scale, area- and power-efficient artificial brain-inspired computational systems.

The key benefit of RRAM scalability is typically leveraged by implementing RRAM cells as crossbar arrays; a configuration that maximises memory area density (down to $4 F^{2} /$ storage node for planar crossbars, where $F$ is the minimum feature size of the crossbar array [6], [7]). The main drawback of this implementation, however, is the issue of 'sneak currents' [8] whereby current tends to pass through

Alexander Serb, William Redman-White and Themis Prodromakis are with the Southampton Nano Group based in Nano-Fabrication Centre, Department of Electronics and Computer Science, Faculty of Physical Science \& Engineering, University of Southampton, Highfield, Southampton SO17 1BJ. Christos Papavassiliou is with the Department of Electrical and Electronic Engineering Department, Imperial College London, SW7 2AZ.

Corresponding author e-mail: A.Serb@soton.ac.uk. Other author emails: \{wrw, T.Prodromakis\}@soton.ac.uk, \{c.papavas\}@imperial.ac.uk respectively. \footnotetext{
switching, resistive state drift etc.).
}

Copyright (c) 2015 IEEE. Personal use of this material is permitted. However, permission to use this material for any other purposes must be obtained from the IEEE by sending an email to pubs-permissions@ieee.org.

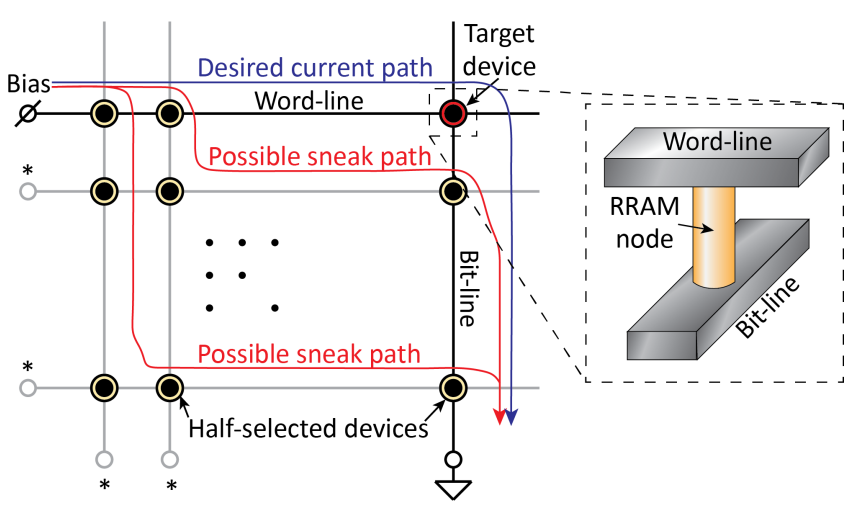

Fig. 1. The crossbar sneak current problem: when attempting to interface a target device 'sneak current' will flow through 'sneak paths', thus corrupting the accuracy of read operations and potentially disrupting non-target devices during the write operation. The specific paths and magnitudes of sneak currents will depend on the biasing regime applied to the nodes marked as '*'. Inset shows the structure of each cross-point.

devices other than the device under test (DUT) during 'read' and 'write' operations (Fig. 1). Sneak currents prove disruptive to the accuracy of read operations [9] and may precipitate programming of non-target storage nodes during write operations.

Research towards mitigating sneak current effects revolves mainly around the development of 'selector devices' that can be embedded into the storage nodes themselves and allow highly selective targeting of DUTs [10]-[12]. The implementation of selector devices, however, adds complexity to the overall fabrication process. Sneak currents can be mitigated in selectorless arrays too by e.g. employing suitable biasing regimes (for overview of such biasing regimes see section 2.6 in [13]) or attempting to calculate cross-point resistance via multiple, multi-port measurements [14]. Each mitigation/readout strategy has merits and drawbacks.

In this paper we build upon previous work [15] and investigate the readout accuracy limits in selectorless, multi-level RRAM crossbar arrays for three distinct read-out techniques. We implement them on a custom-built instrumentation platform and compare their ability to successfully read resistive states from a small $(12 \times 12)$, selectorless reference array consisting of linear resistors. We then further broaden the scope of our study by extrapolating towards the scaling limits of RRAM selectorless arrays as well as worst-case scenarios via SPICE simulations. The focus is kept on well-behaved linear test devices in order to eliminate any sources of uncertainty that would be present if RRAM devices had been used (inadvertent 
The capability of performing accurate array-level read-outs is a crucial element in many applications. One example is the mass testing of novel device architectures, where array-level electrical characterisation would be a major boost towards the automation of the process development cycle. Another example can be found in multi-level memory cell development, where the amount of information potentially extractable from storage nodes able to assume resistive states within a continuous range is read-out accuracy-limited. Finally, the field of neuromorphic engineering could exploit the benefits arising from the development of nanoelectronic artificial synapse banks far more efficiently if there was an option to monitor the internal state of every synapse in the bank for debugging and analysis purposes.

The paper is organised as follows: Section II provides the definition of 'memory state' and introduces the three different read-out techniques. Section III presents a physical system capable of performing all three types of read operation whilst section IV provides measured results from our prototyped reference array used to assess the instrument's accuracy. Section $\mathrm{V}$ presents SPICE simulations whereby system performance is estimated for larger arrays set up in standard 'worst-case' configurations. Finally, section VI discusses the merits of each read operation technique in light of the simulated and measured results and draws the overall conclusions of the paper.

\section{THEORETICAL BACKGROUND}

\section{A. Defining a memory state}

The resistive state (RS) of any two-terminal, non-linear component (such as typical RRAM devices) can be measured in a multitude of ways, e.g. as the static or differential resistance as measured when some fixed potential difference $(\Delta V)$ is applied across the component. In practice it is convenient to use the static resistance at fixed $\Delta V$ definition. This approach is amenable to easy circuit implementation as it employs a constant read-out voltage and allows for the determination of DUT RS via a simple amperometric measurement. In this work, we define RS as static resistance measured at $\Delta V=0.5 \mathrm{~V}$. This value was arbitrarily chosen in order to demonstrate sub-volt operation whilst allowing our instrumentation platform to operate well above the noise floor. Read-out voltage optimisation lies outside the scope of this paper.

\section{B. Crossbar array nomenclature}

Reading from a crossbar array typically involves targeting a single cross-point element at a time and attempting to determine its resistive state. This gives rise to 'active' (leading to the target device) and 'inactive' word- and bit-lines. In order to simplify the tangled structure of the crossbar network, read-out operations are typically performed with all inactive word-lines shorted together and similarly all inactive bitlines shorted together. This reduces the crossbar network to a 'four-node-four-component' $(4 \mathrm{n} 4 \mathrm{c})$ system where the four components are: 1) the target device alone $\left.\left(R_{T}\right), 2\right)$ the devices sharing word-line with the target (we shall call this the "word

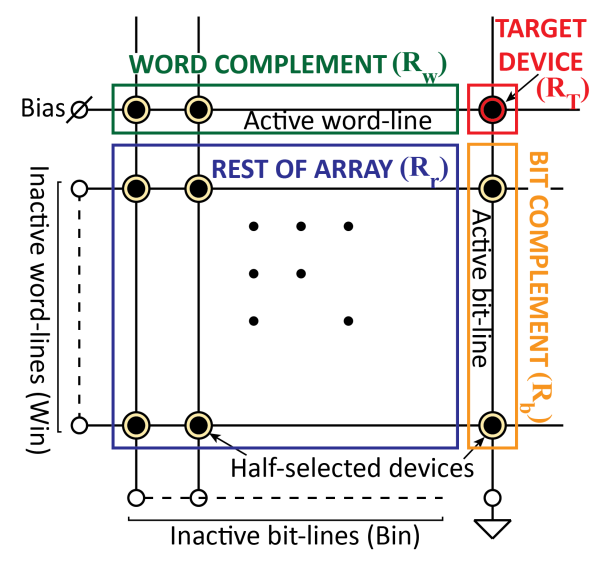

(a)

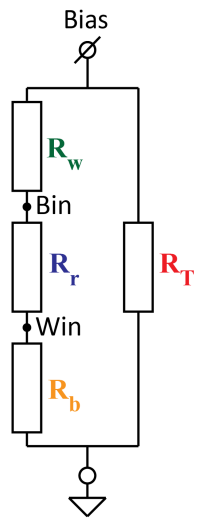

(b)
Fig. 2. Crossbar array terminology: (a) Once a device has been selected for a read or write operation the array is split into four sub-arrays and the word- and bit-lines are classified as active or inactive depending on whether they lead to the target device or not. (b) ' $4 n 4 c$ ' approximation of array when inactive word- and bit-lines are shorted together.

complement' of $R_{T}$ ) with equivalent resistance $\left.R_{w}, 3\right)$ the devices sharing bit-line with the target (the 'bit complement' of $R_{T}$ ) with equivalent resistance $R_{b}$ and 4) the rest of the array with equivalent resistance $R_{r}$ (see [9], [14]). Fig. 2 illustrates these concepts. We shall use this reduced system in order to describe how the three read-out operations function conceptually.

\section{The three read-out techniques}

1) Single read - one direct measurement: There are many biasing regimes that can be used to carry out a 'single read' operation. The specific choice of regime will depend largely on the type of device being used (e.g. unipolar- vs. bipolarswitched) and other considerations, such as power dissipation, but ultimately it will aim to simultaneously determine the voltage drop across, and the current through the target device:

$$
R_{T}=\frac{V_{b}}{i_{T}}
$$

where $R_{T}$ is the target resistance to be determined, $V_{b}$ denotes the standard read-out voltage applied across the target device (in this work $V_{b}=0.5 \mathrm{~V}$ ) and $i_{T}$ is the current through the target device. $i_{T}$ can be determined by bootstrapping the wordor bit-complement devices (or both) and measuring current entering/exiting the active line servicing the bootstrapped devices. An example of a possible single read measurement configuration is shown in Fig. 3(a). A good way to implement it in practice would be by realising the ammeter as a transresistance amplifier (TRA), thus enforcing virtual earthing of the active bit-line. This, in turn, ensures bootstrapping of the bit-complement whilst keeping the voltage drop across the target device under control as shown in Fig. 3(b).

This technique is relatively easy to implement and relies on a single measurement, but when the target device is in very high RS, $i_{T}$ becomes very small. This generates vulnerability to systematic offset error factors such as constant leakage currents and especially voltage offsets that affect the quality 


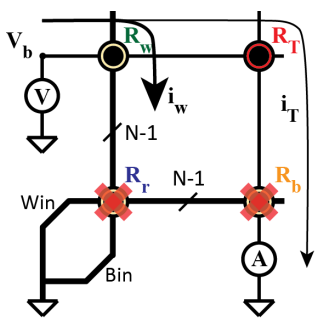

(a)

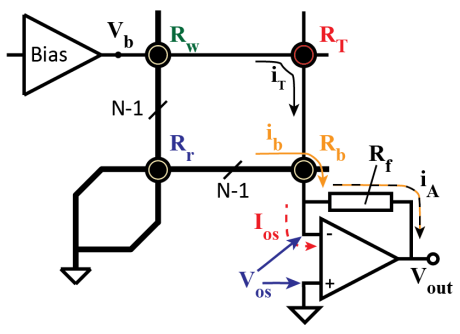

(b)
Fig. 3. Example of a 'single read' operation: (a) Ideal concept where the voltage source provides a known bias, whilst the ammeter measures the current through the target device $i_{T}$. A significant amount of current may pass through the word-complement $\left(i_{w}\right)$ towards GND. $R_{b}$ is bootstrapped in this example while $R_{r}$ is shunted. (b) Possible circuit implementation where a trans-resistance amplifier (TRA) plays the role of the ammeter. Amplifier offset voltage $\left(V_{o s}\right)$ and current $\left(I_{O S}\right)$ are shown explicitly.

of bootstrapping as seen in Fig. 3(b). The importance of proper bootstrapping for the correct operation of the single read technique can be demonstrated by calculating the current reaching the ammeter in the example of figure 3(b) if amplifier offset voltage $V_{o s} \neq 0$ and offset current $I_{o s}$ small:

$i_{A}=i_{T}+i_{b}=\frac{V_{b}-V_{o s}}{R_{T}}-\frac{V_{o s}}{R_{b}\left(V_{o s}\right)} \approx \frac{V_{b}}{R_{T}}-\frac{V_{o s}}{R_{b}\left(V_{o s}\right)}$

where $i_{A}$ is the current through the ammeter, $i_{b}$ the current through the $(N-1)$ devices constituting $R_{b}, R_{b}\left(V_{o s}\right)$ the static resistance of the $R_{b}$ component at a bias voltage of $V_{o s}$ and $V_{o s} \ll V_{b}$ in the approximated r.h.s. If $V_{o s}$ is small, then the magnitudes of $i_{T}$ and $i_{b}$ become comparable when $\frac{V_{b}}{V_{o s}}=\frac{R_{T}}{R_{b}}$, which can impose severe constraints on the choice of $V_{b}$ if $R_{b}$ is significantly lower than $R_{T}$. This would be the case when $R_{T}$ is in high RS and $R_{b}\left(V_{o s}\right)$ low.

2) Differential read - two direct measurements: The differential read technique consists of two sub-operations: a standard single read operation under bias voltage $V_{b, 1}=V_{b}=0.5 \mathrm{~V}$ and an additional single read operation performed under zero bias voltage $V_{b, 2}=0 \mathrm{~V}$. Computed target resistance for a differential read operation is given by:

$$
R_{T}=\frac{V_{b, 1}-V_{b, 2}}{i_{t, 1}-i_{t, 2}}=\frac{V_{b}}{i_{t, 1}-i_{t, 2}}
$$

where $i_{t, 1}, i_{t, 2}$ is the current through $R_{T}$ during the first and second sub-operations respectively. $i_{t, 2}$ will compensate $i_{t, 1}$ for systematic offsets present in the system.

The differential read technique computes static resistance at $V_{b}=0.5 \mathrm{~V}$ independent of the shape of the target device $\mathrm{I}-\mathrm{V}$ characteristic only for $V_{b, 2}=0 \mathrm{~V}$. Any other choice of $V_{b, 2}$ causes the technique to yield the $\Delta V / \Delta I$ slope defined by the two chosen voltage bias points along the I-V of the target device. If we set $V_{b, 1} \approx V_{b, 2}$, the differential technique attempts to compute differential resistance at the chosen bias point $\frac{\partial V}{\partial I}\left(V_{b}\right)$.

The main benefit of a 'differential read' approach is its offset cancelling nature, which becomes apparent only when systematic offsets become significant sources of error, overpowering sources of random error such as instrumentation

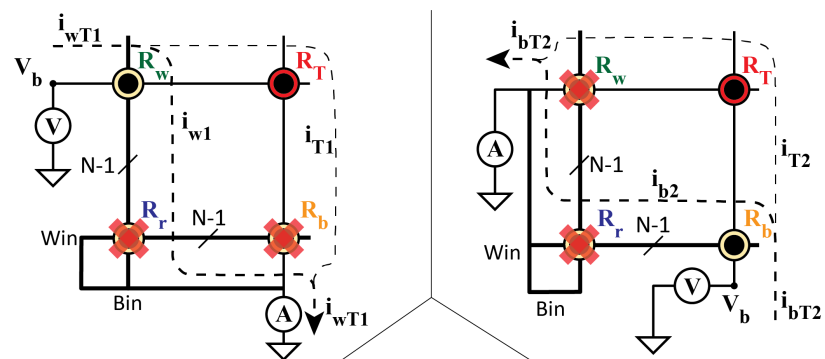

(a)

(b)

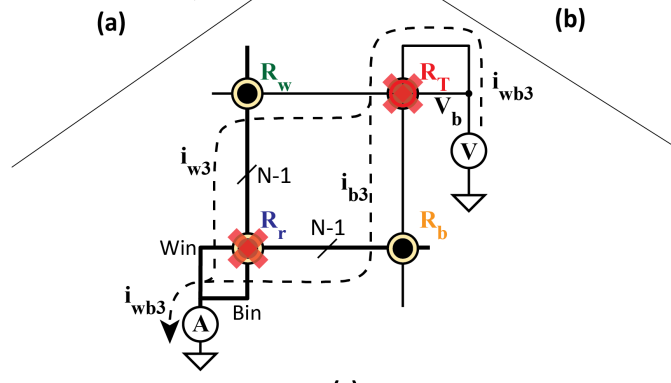

(c)

Fig. 4. Conceptual example of triple read operation: (a) Step 1: determine $R_{w} \| R_{T}$. (b) Step 2: determine $R_{b} \| R_{T}$. (c) Step 3: determine $R_{w} \| R_{b}$ The entire cross-bar is considered as a two-terminal black box throughout the operation. The very low resistance component $R_{r}$ is always shunted for power consumption reasons.

noise. However, the technique requires two sub-operations, one of which is a single read operation; therefore, it is slower than the single read technique.

3) Triple read - three proxy measurements: The 'triple read' operation is based upon the idea of computing $R_{T}$ by proxy. The procedure flows as follows (see Fig. 4): a) Measure the equivalent resistance of all devices in the target word-line (the 'full word' with equivalent resistance $R_{w T}=R_{w} \| R_{T}$ ). b) Measure the resistance of the target bit-line ('full bit', $\left.R_{b T}=R_{b} \| R_{T}\right)$. c) Short together and bias the active wordand bit-lines with the aim of shunting $R_{T}$ whilst determining the 'full complement', $R_{w b}=R_{w} \| R_{b}$. The procedure yields three equations for three unknowns and hence $R_{T}$ can be computed, as shown in [14]. All read-outs are performed at the standard $V_{b}=0.5 \mathrm{~V}$.

The equations for each sub-operation are as follows:

$$
\begin{gathered}
R_{w T}=\frac{V_{b}}{i_{w T, 1}} \\
R_{b T}=\frac{V_{b}}{i_{b T, 2}} \\
R_{w b}=\frac{V_{b}}{i_{w b, 3}}
\end{gathered}
$$

where $i_{x, y}$ indicates current through $R_{x}$ during the $y$ th suboperation.

It then follows that:

$$
G_{T}=\frac{G_{w T}+G_{b T}-G_{w b}}{2} \Rightarrow R_{T}=\frac{2 V_{b}}{i_{w T, 1}+i_{b T, 2}-i_{w b, 3}}
$$


where $G_{x}=\frac{1}{R_{x}}$.

Although algebraically a correct method for determining $R_{T}$, this method suffers from an inherent tendency to amplify errors committed while determining its partial results $R_{b T}, R_{w T}$ and $R_{w b}$. Let us define the fractional read-out error of any single read measurement $F$ as:

$$
F=\frac{R_{\text {meas }}-R_{\text {nom }}}{R_{\text {nom }}}
$$

where $R_{\text {nom }}$ is the nominal and $R_{\text {meas }}$ the measured RS of the target element(s). Noting that $G_{x y}=G_{x}+G_{y}$ it can be show that computed target device conductance $G_{T, \text { meas }}$ in the presence of read-out errors is given by:

$$
\begin{aligned}
2 \cdot G_{T, \text { meas }}= & (1+\alpha)\left(G_{w}+G_{T}\right)+(1+\beta)\left(G_{b}+G_{T}\right) \\
& -(1+\gamma)\left(G_{w}+G_{b}\right)
\end{aligned}
$$

where $\alpha, \beta, \gamma$ are the fractional errors in reading $G_{w T}, G_{b T}$ and $G_{w b}$ respectively and $G_{T}$ the actual target device conductance.

The fractional error in determining $G_{T}$ is then given by:

$$
\frac{G_{T, \text { meas }}-G_{T}}{G_{T}}=\frac{\alpha+\beta}{2}+\frac{(\alpha-\gamma) G_{w}}{2 \cdot G_{T}}+\frac{(\beta-\gamma) G_{b}}{2 \cdot G_{T}}
$$

Eq. 10 shows that the fractional error involved in reading $G_{w T}$ and $G_{b T}$ transmits directly into the fractional error in reading $G_{T}$, but the terms $(\alpha-\gamma)$ and $(\beta-\gamma)$ are amplified by $\frac{G_{w}}{G_{T}}$ and $\frac{G_{b}}{G_{T}}$ respectively. Furthermore, the Bienayme formula ${ }^{1}$ and the variance scaling property ${ }^{2}$ tell us that any variance in $\alpha, \beta$ and $\gamma$ (random read-out errors e.g. due to noise in the circuitry) will be amplified by $\frac{G_{w}}{G_{T}} / \frac{G_{b}}{G_{T}}$ and add-up to cause higher final computed $R_{T}$ variance vs. variance in measurements of $R_{w T}, R_{b T}$ and $R_{w b}$.

Theoretically, the main benefit of using this technique would be that the entire crossbar array is treated as a two-terminal device in every sub-operation. This implies that unlike in single and differential read-out, all current entering the array will exit through the ammeter, whilst the exclusive use of shunting (as opposed to bootstrapping) in order to neutralise various components of the crossbar (e.g. $R_{b}$ or $R_{w}$ ) should remove the requirement for careful handling of offsets. However, this technique requires three sub-operations to complete and the ability of the system to treat each crossbar line as either a word- or a bit-line flexibly. The relatively minor increase in circuit complexity needed to accommodate this additional flexibility is overshadowed by the fact that during the last sub-operation current flows through both $R_{w}$ and $R_{b}$, but in opposite polarities from the perspective of the DUTs. Therefore, this read-out technique is not suitable for arrays consisting of storage nodes with asymmetric I-V curves.

As a side-note we notice a further benefit of this technique concerning speed: Each sub-operation aims to compute a low resistance formed by the parallel combination of either $N$ or $(2 N-1)$ devices. Consequently, for sufficiently large ' $\mathrm{N}$ ' the determination of all 1.h.s. terms in equations 4,5 and

\footnotetext{
${ }^{1}$ The variance of the sum or difference of $n$ uncorrelated random variables equals the sum of their $n$ individual variances.

${ }^{2}$ The variance of $k \cdot X$, where $X$ is a random variable and $k$ a constant, equals $k$ times the variance of $X$.
}

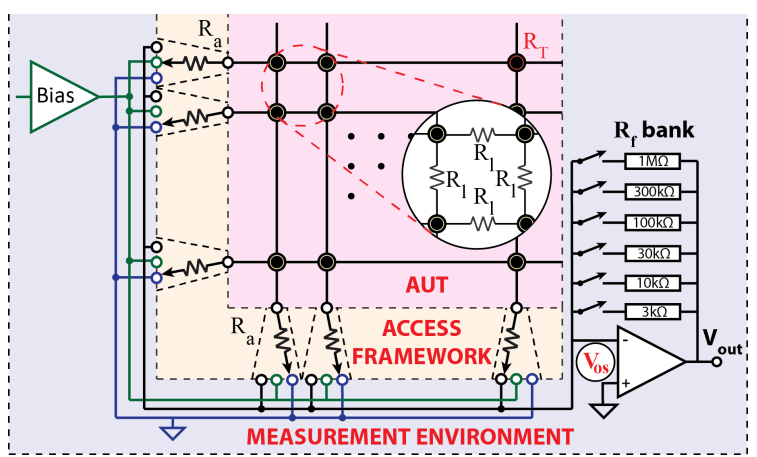

Fig. 5. Simplified instrumentation platform schematic showing the 'Array Under Test' (AUT, pink), the access framework (yellow) and the measurement environment (blue). Key sources of error are also shown: access resistance $R_{a}$, line resistance $R_{l}$ and TRA offset voltage $V_{o s}$.

6 can potentially be much faster than obtaining a 'single' or 'differential' read result. This speed benefit will tend to improve with increased array size. The detailed study of the transient behaviour of the three read-out techniques is outside the scope of this paper where we concentrate on steady-state behaviour (read-outs are assumed to be taken when all voltages throughout the system have settled).

\section{SYSTEM IMPLEMENTATION}

Fig. 5 shows a simplified schematic of the instrumentation platform used to implement the read-out techniques under study. At the core sits the crossbar array under study. Around it lies the access framework module, which consists of singlepole-triple-throw (SP3T) switches whose positions determine which lines act as active and inactive word- and bit-lines. Pairs of relays (G6EU-134P-US) were used in order to implement the SP3T functionality whilst minimising access resistance ( $R_{a}-50 \mathrm{~m} \Omega$ max.). Finally, the measurement environment consists mainly of the bias generator and the TRA. The TRA consists of a precision OpAmp (OPA227 - $\pm 200 \mu V$ max. offset) and its feedback resistor $\left(R_{f}\right)$ bank (precision resistors - $5 / 6$ resistors at $0.1 \%$ tol. $1 / 6$ at $1 \%$ ) and acts as an ammeter with output voltage $V_{\text {out }}$ as its current-reading variable. The $R_{f}$ bank allows the TRA to measure a large range of currents whilst maintaining $V_{\text {out }}$ within the amplifier's output swing limits. Each resistor in the $R_{f}$ bank is software-assigned to measure target loads within given RS ranges (table I). The boundaries between the RS ranges of adjacent $R_{f}$ resistors are given by their geometric means. The bias generator consists of an LT1970A amplifier whose output is measured each time a device is read in order to improve measurement accuracy.

At higher level the system is operated by a microcontroller (mBED LPC1768) and implemented on a custom-made PCB with discrete components. This is crucial as it allowed us to utilise very high spec components in order to test the limits of read-out accuracy. Other system modules, not shown in the schematic of Fig. 5, include: read-out buffers, voltage references, the power management unit and the device programming unit. A photograph of the instrument is shown in Fig. 6. 
TABLE I

TARGET LOAD RS CLASSES.

\begin{tabular}{c|cccc} 
Class ID & $R_{f}(k \Omega)$ & Min. RS $(k \Omega)$ & Max. RS $(k \Omega)$ & $n^{*}$ \\
\hline 1 & 3 & 0 & 5.48 & 28 \\
2 & 10 & 5.48 & 17.3 & 50 \\
3 & 30 & 17.3 & 54.7 & 39 \\
4 & 100 & 54.7 & 173 & 25 \\
5 & 300 & 173 & 547 & 2 \\
6 & 1000 & 547 & $\infty$ & 0
\end{tabular}

* Number of devices in reference crossbar (see section IV).

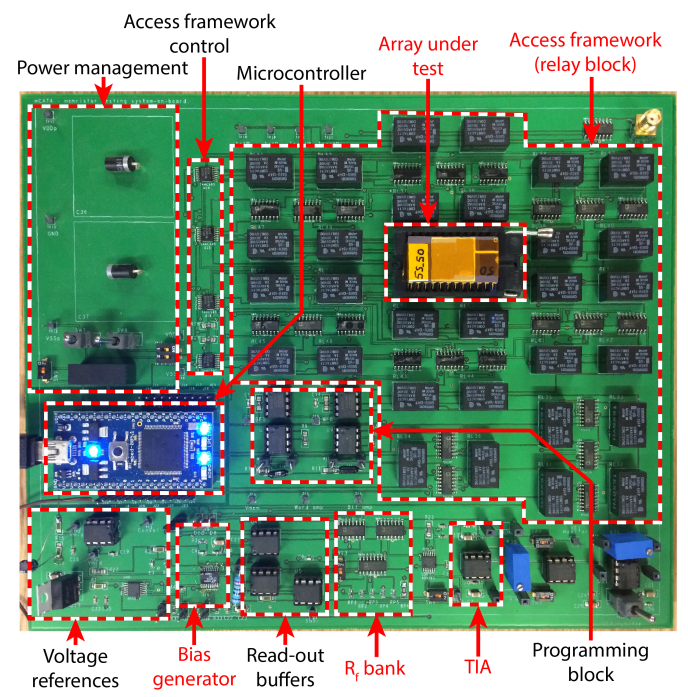

Fig. 6. Photograph of PCB-based instrumentation platform implementing the read-out techniques under study. Blocks referred to in Fig. 5 are labelled in red.

\section{EXPERIMENTAL RESULTS}

The performance of the 'single', 'differential' and 'triple' read-out techniques was benchmarked against a reference crossbar array. The array consisted of discrete, linear resistors with RS ranging between $1 k \Omega$ and $220 k \Omega$ (Fig. 7); values covering the initially intended region of operation of the instrument $(1 k \Omega-100 k \Omega)$. Notable features of the reference array include: a) An all-low RS ( $1 k \Omega$ ) bit-line intended to uncover the effects of attempting to bootstrap a very low RS path. b) A high RS $(>100 k \Omega$ ) word-line testing read-out performance at excessively high RS. c) Components randomly drawn from pots of available devices to provide insight concerning read-out of intermediate-value components in a relatively homogeneous environment.

Every device in the reference crossbar was measured with each technique described in section II and the fractional readout error was computed. Results obtained from measurements on the reference array are shown in Fig. 8. Notably, results for the single and differential read-out techniques are very similar and cover broadly similar ranges in terms of $F$. On the other hand, results obtained for the triple read show fractional errors up to thousands of percentage points above and below nominal. This is rather surprising considering that the partial results for full word, full bit and full complement are all fairly tightly distributed.

Panels (d) and (e) in figure 8 show characteristic horizontal,

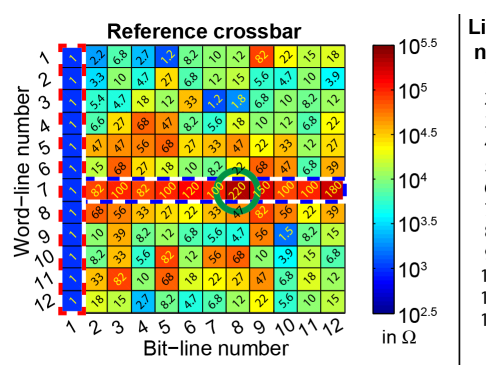

(a)

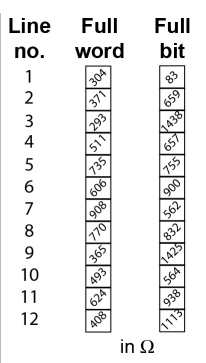

(b)

Fig. 7. Reference resistor crossbar array used for assessing read-out quality: (a) Resistor configuration. Numbers at each cross-point location indicate RS in $k \Omega$. White/red dashed line: all-low RS bit-line. White/blue dashed line: high RS word-line. Green-circled device: $220 \mathrm{k} \Omega \mathrm{RS}$; the highest value in the reference array. Numbers in each cell indicate device resistance in $k \Omega$. (b) Full word and full bit resistances. Numbers in each box are in $\Omega$.

respectively vertical bands. This is because the triple read suboperations used to determine the full word or full bit RS are procedurally identical and therefore results should not depend on the specific selection of target device. As a result, panels (d) and (e) of Fig. 8 contain 12 independent measurements of each full word and full bit RS; one for each device in a line. Differences within each set of 12 measurements reveal the effects of random measurement errors. These effects are summarised in table II where for each word/bit line, average fractional error $\bar{F}$ over all 12 measurements and corresponding standard deviation $(\sigma)$ were computed. $\sigma$ can be a useful indicator of spread in the data even though the underlying distribution may not necessarily be Gaussian.

TABLE II

AVERAGE FULL WORD AND FULL BIT MEASUREMENT FRACTIONAL ERROR $\bar{F}$ AND STANDARD DEVIATION $\sigma$ BY WORD-/BIT-LINE IN $\%$.

\begin{tabular}{c|cc|cc} 
& \multicolumn{2}{|c|}{ Full word } & \multicolumn{2}{c}{ Full bit } \\
\hline Line number & $\bar{F}$ & $\sigma$ & $\bar{F}$ & $\sigma$ \\
\hline 1 & 2.506 & 0.188 & 3.571 & 0.117 \\
2 & 2.718 & 0.168 & 4.483 & 0.223 \\
3 & 2.392 & 0.117 & 6.292 & 0.229 \\
4 & 3.428 & 0.318 & 3.799 & 0.218 \\
5 & 3.927 & 0.269 & 3.752 & 0.149 \\
6 & 3.559 & 0.219 & 4.033 & 0.143 \\
7 & 3.798 & 0.299 & 3.354 & 0.194 \\
8 & 3.756 & 0.167 & 4.202 & 0.213 \\
9 & 2.191 & 0.189 & 6.263 & 0.394 \\
10 & 3.071 & 0.253 & 3.023 & 0.224 \\
11 & 3.490 & 0.232 & 4.017 & 0.294 \\
12 & 2.714 & 0.214 & 4.524 & 0.266 \\
\hline Average & $\mathbf{3 . 1 2 9}$ & $\mathbf{0 . 2 1 9}$ & $\mathbf{4 . 2 7 6}$ & $\mathbf{0 . 2 2 2}$
\end{tabular}

Many pairs of lines are read with statistically significant fractional error differences $(\Delta \bar{F}$ significantly larger than both associated standard deviations $\sigma$ ) as can be seen by examining e.g. the cells highlighted in yellow in table II (two-tailed twomeans t-test shows values of $\bar{F}$ are significantly different with $\mathrm{p}$-value $\approx 0.00095$ ). This indicates that each line is read at a fundamentally distinct fractional error; possibly a function of the RS of the line itself and/or the state of the rest of the crossbar array. This is important because it implies that the fractional errors committed in measuring the terms in the 1.h.s. of eq. 7 do not necessarily cancel each other out. At a higher level we also observe that our reference array's word- and 

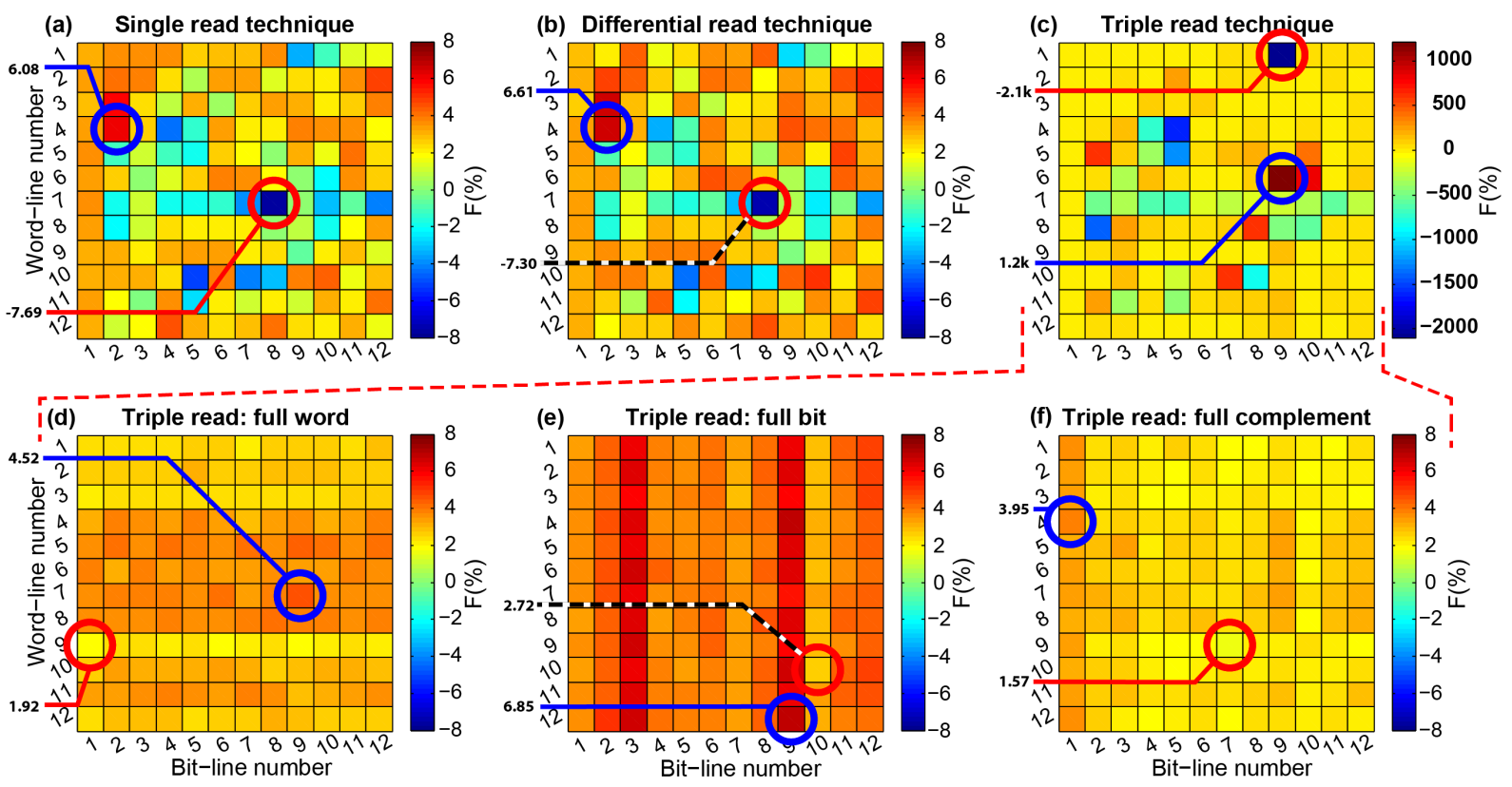

Fig. 8. Fractional read-out errors $F$ obtained by reading the reference crossbar array: (a-c) Results for single, differential and triple read-out techniiques. (d-f) Partial results that combine through eq. (7) to yield panel (c). Devices showing maximum and minimum errors are circled in blue or red circles respectively. Note that all panels except (c) have been plotted vs. the same colour-bar range.

bit-lines are read at significantly different average fractional errors although for both each line contains readings with, on average, similar spread.

Revisiting eq. 10 we note that the $\frac{G_{w}}{G_{T}}$ and $\frac{G_{b}}{G_{T}}$ factors take large values for some devices, as evidenced in Fig. 7: for example the device sitting at (word, bit) location $(7,8)$ has a value of $220 \mathrm{k} \Omega$ whilst its corresponding full word and full bit have values of $908 \Omega$ and $832 \Omega$ respectively. If we set device $(7,8)$ as the target, then we obtain $\frac{G_{w}}{G_{T}}=\frac{R_{T}}{R_{w}} \approx 242.3$ and $\frac{R_{T}}{R_{b}} \approx 264.4$. Even in the absence of systematic fractional errors $\bar{F}=0$, this would lead relatively small amounts of random error in determining $\alpha, \beta$ and $\gamma$ (see $\sigma$ values in table II) to generate intolerably high read-out errors on most trials.

In order to investigate the resulting data further, the distributions of fractional errors and their corresponding cumulative distributions were extracted for each read-out technique as shown in Fig. 9, top two rows. The bottom row shows cumulative error distributions separately for devices belonging to each RS class. Notably, both single and differential readout show a propensity for overestimating smaller resistances whilst underestimating larger ones. In both cases the worst performers tend to be the devices in RS class 5 . The relatively large number of devices in RS classes 1 and 2 skews the overall error distribution towards $\mathrm{a} \approx 3 \%$ average overestimate. In the triple read case, the error distribution shows a vast range of values, including many resistances that were read as negative $\left(F<-100 \%\right.$ implies $\left.R_{\text {meas }}<0\right)$. Devices exhibiting higher RS tend to suffer much higher read-out errors.

\section{SPICE ANALYSIS}

In order to investigate how the three read-out techniques can be expected to perform in the presence of a controlled amount of realistic non-idealities, each was examined through SPICE simulations based on the simplified system schematic in fig. 5. Table III shows the list of non-ideality factors taken into account, of which $R_{l}, R_{a}$ and $V_{o s}$ were of key significance. All non-ideality factors except $R_{l}$ were based on the components used in our system and kept fixed for all simulations. The TRA core amplifier was modelled behaviourally, although result accuracy was validated with the more computationally demanding SPICE model provided by the supplier of the component (Texas Instruments).

\section{TABLE III}

VALUES OF MEASUREMENT INSTRUMENT AND CROSSBAR ARRAY NON-IDEALITY FACTORS USED IN SIMULATIONS SHOWN IN THIS WORK.

\begin{tabular}{l|ccc} 
Parameter & Symbol & Value & Units \\
\hline Line resistance & $R_{l}$ & $50^{\dagger}-10600^{\ddagger}$ & $m \Omega$ \\
Access resistance & $R_{a}$ & $300^{*}$ & $m \Omega$ \\
TRA offset voltage & $V_{o s}$ & $-10^{\S}$ & $\mu V$ \\
\hline TRA output impedance & $R_{T R A}$ & $100^{\S}$ & $\mu \Omega$ \\
TRA open loop DC gain & AOL & $10^{8 \S}$ & - \\
$\dagger$ Conservative theoretical calculation for strip-board line resis- \\
tance. \\
Theoretical calculation for $100 \times 10 \mathrm{~nm}$ cross-section $\mathrm{Pt}$ \\
electrode in a $100 \mathrm{~nm}$ pitch crossbar. \\
* G6EU-134P-US relay datasheet and multimeter readings. \\
§ OPA227 datasheet typ. value.
\end{tabular}

The simulated system was first tested with the reference array from fig. 7 in order to compare performance against the physical system and then with a $12 \times 12$ 'worst-case' array with $R_{O N}=1 \mathrm{k} \Omega$ and $R_{O F F}=100 \mathrm{k} \Omega . R_{l}$ was $50 \mathrm{~m} \Omega$ in both cases. We define 'worst-case' arrays as $N \times N$ arrays consisting of linear I-V elements where the device farthest from the access resistors, in our case devices with (word,bit) coordinates of $(1, \mathrm{~N})$, is at the maximum allowed RS $\left(R_{O F F}\right)$ whilst every other cross-point node is set at the minimum RS 
SINGLE READ
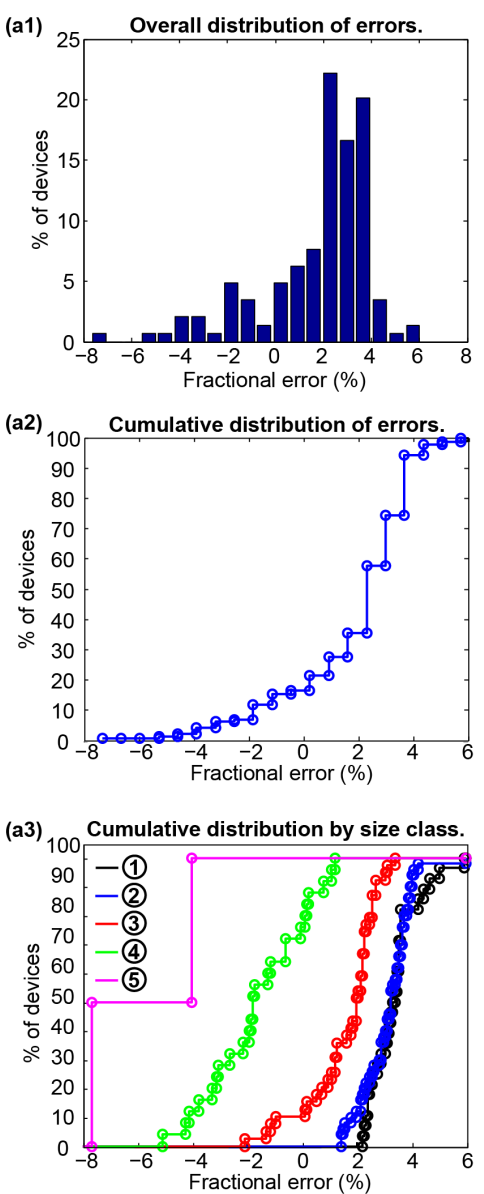

DIFFERENTIAL READ
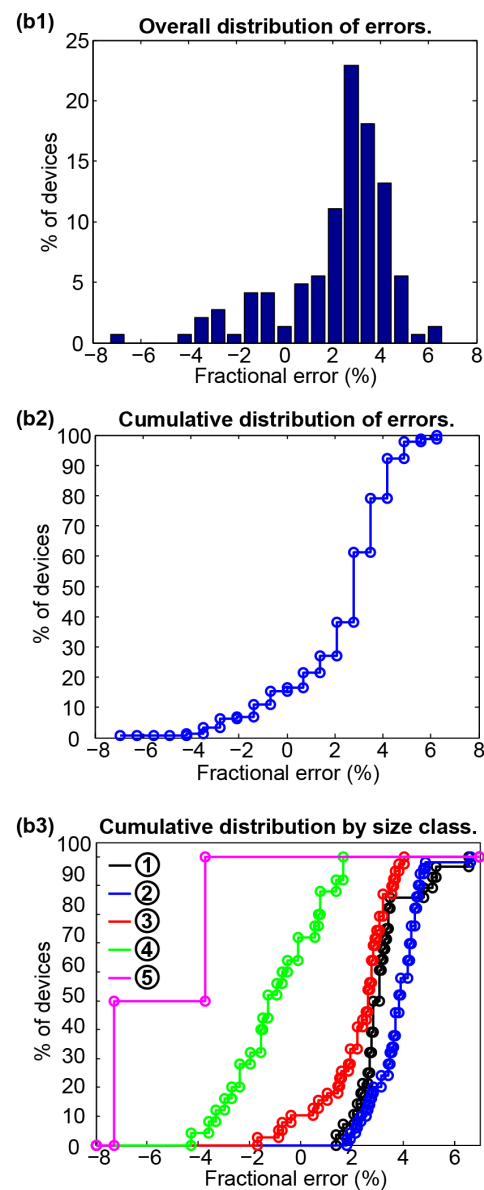

TRIPLE READ

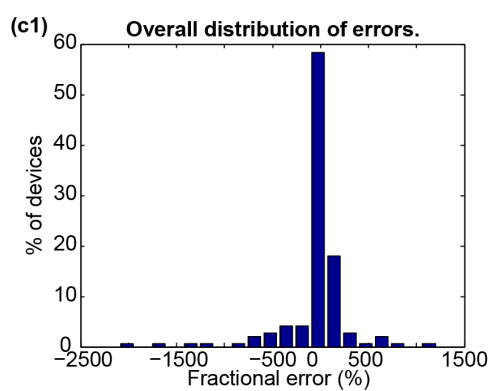

(c2)
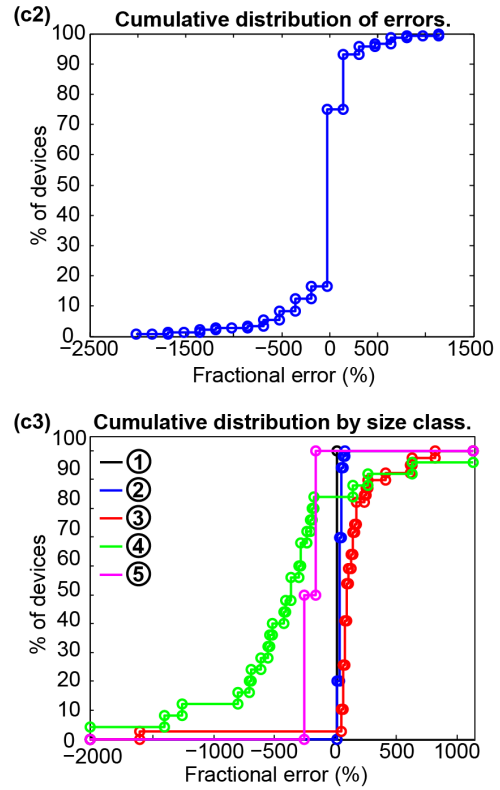

Fig. 9. Fractional read-out error distributions for measurements obtained employing (a) single, (b) differential and (c) triple read-out techniques. Absolute distributions (top row), cumulative distributions (middle row) and cumulative distributions by RS class (bottom row) are shown. Circled numbers in the bottom row map plot colours to RS class.

$\left(R_{O F F}\right)$-see fig. 10(d)-. Results are summarised in fig. 10.

Results on the reference array show that under the simulated system set-up the low RS bit-line exhibits significantly higher errors than the rest of the array due to the effects of line resistance. This is confirmed by noting that as one moves closer towards the bit-line access switch (towards word-line 12) the errors abate in a gradual fashion. This issue affects all read-out techniques similarly. Next, we notice that only in the case of the single read we can still observe traces of the pattern present in fig. 8(a). This pattern vanishes when the single read operation is simulated with an offset-free TRA. The differential and triple read techniques seem to eliminate much of the propensity of the single read technique to underestimate devices at high RS, likely because of the offset-cancelling nature of the differential read.

Results on the $12 \times 12$ worst-case array confirm that the differential and triple reads mitigate high RS device underestimation, as seen in the table inset in fig. 10(d). In the case of the triple read the $R_{O F F}$ device is read successfully because word- and bit-complements are read with exactly the same $F \approx 0.5881 \%$ whilst the full complement is read at an extremely close $F \approx 0.5880 \%$. The main contribution to the final overall read-out $F$ of $0.73 \%$ comes from the first r.h.s term of eq. $(10)(\approx 0.59 \%)$.

Interestingly, in this particular array set-up it is the worst of the $R_{O N}$ devices that forms the bottleneck of the design. Worst $R_{O N}$ shows a fairly consistent $F \approx+1.35 \%$ and is located at address $(2,11)$ for all read-out techniques (marked in a red box in fig. 10(d)). Notably, this is the device farthest from the word- and bit-line access switches that does not have an $R_{O F F}$ device on either of its lines; results underlining that the worst performing device is not always the most obvious one (typically assumed to be the high RS device).

Next, simulations were carried out in a variety of worstcase arrays of different sizes and $R_{l} . R_{l}$ was swept between a minimum of $50 \mathrm{~m} \Omega$ corresponding to expected line resistance for the reference array and a maximum of $10.6 \Omega$, corresponding to expected line resistance for an array employing $\mathrm{Pt}$ electrodes with $100 \times 10 \mathrm{~nm}$ cross-sectional area and $100 \mathrm{~nm}$ pitch. The fractional read-out errors for the high RS device are shown in figure 11.

Read-out accuracy is very similar for all read-out techniques indicating that line resistance and overall system loading affect all three read-out techniques similarly in the idealised simulation framework used in this section. We notice three key trends. First, small arrays with low line resistance tend to 

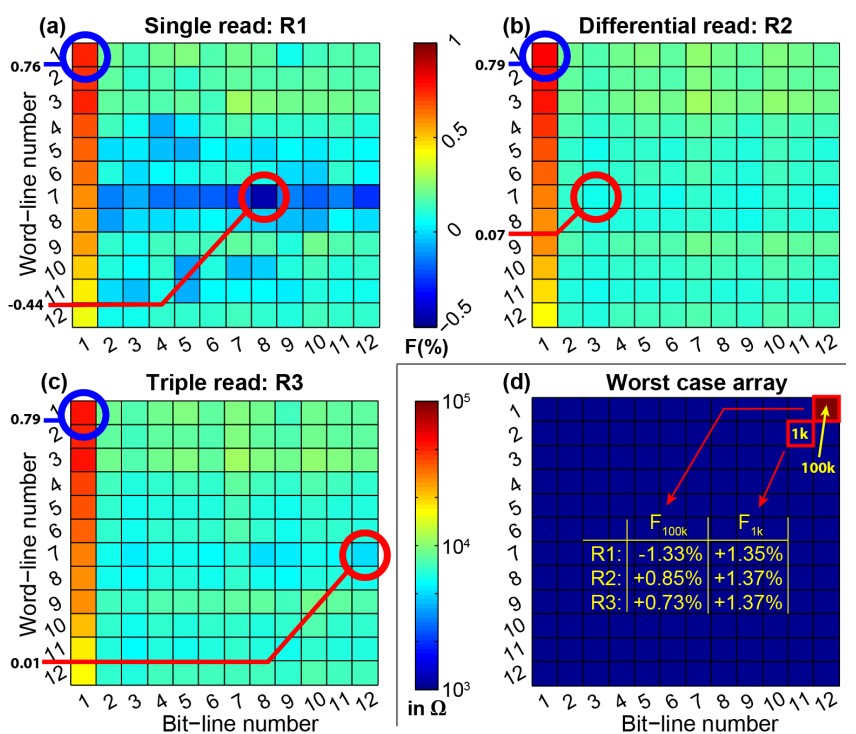

Fig. 10. Performance of simulated crossbar array read-out system (see table III): (a-c) reference crossbar array from fig. 7. Fractional read-out errors $F$ for each read-out technique are shown with devices exhibiting maximum and minimum $F$ marked with blue, respectively red circles; upper colour-bar. (d) Typical 'worst-case' array where all devices are at $R_{O N}=1 k \Omega$ except the device farthest from the word- and bit-line access switches, which is set at $R_{O F F}=100 \mathrm{k} \Omega$; lower colour-bar. Tabulated data inside the array shows $F$ for the $R_{O F F}$ device and the worst of the $R_{O N}$ devices. The $R_{O N}$ device showing the largest $F$ magnitude was found to be the same for all read-out techniques and is marked in a red box.

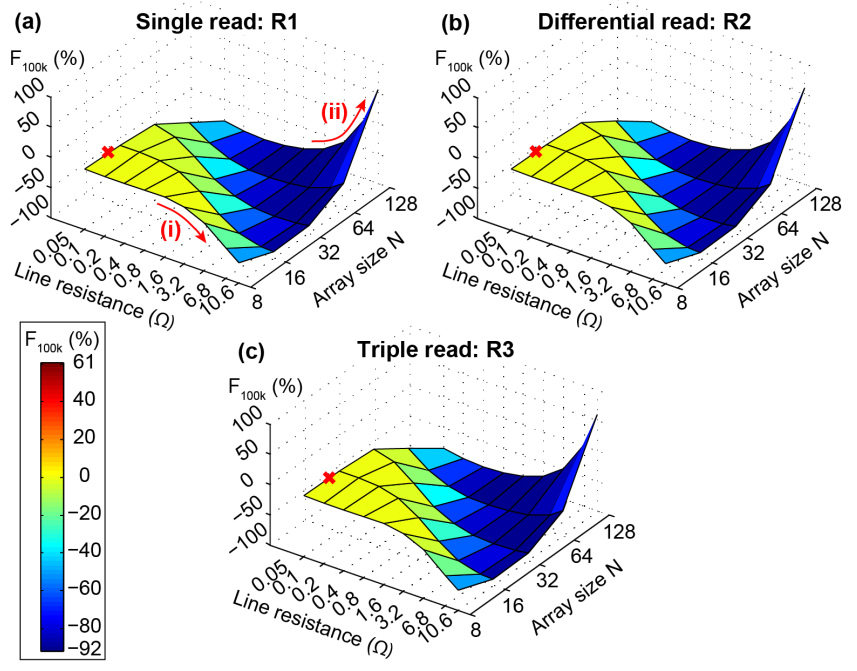

Fig. 11. SPICE-simulated fractional read-out errors of high RS device $\left(F_{100 k}\right)$ in 'worst-case'-configured arrays for: (a) single read, (b) differential read and (c) triple read techniques as a function of array size $(N)$ and line resistance $\left(R_{l}\right)$. The small red crosses indicate the points in the $\left(N, R_{l}\right)$ space for which the values in the inset table of fig. 10(d) were taken. All panels share colour-bar; in panel (a) interesting trends are marked as (i) and (ii) -see text-. allow for accurate read-out of the target device, as expected, but as line resistance increases target resistance starts to be underestimated (fig. 11(a) marked (i)). This is probably caused by the line resistance interfering with the bootstrapping/shunting of the active line(s). For example in fig. 3 the inactive word-lines can no longer be assumed to be sufficiently well grounded throughout their entire lengths, thus resulting in above ground voltages at the word-line terminals of the bitcomplement devices. When the inactive bit-line terminals of the bit-complement devices are below those of their wordline terminals (a situation aided by low $i_{T}$ currents) extra current is injected onto the active bit-line and hence the TRA block overestimates the current through the target element. Secondly, if line resistance continues to grow the system tends to start overestimating target element resistance (fig. 11(a) marked (ii)). This probably occurs because voltage delivery to the target element fails completely, all current between bias generator and grounding effectively by-passing the target element and choosing shorter pathways throughout the array. Finally, we notice that for larger arrays the two aforementioned trends continue to be present, but manifest their presence at lower values of $R_{l}$.

\section{DISCUSSION}

In this paper we have examined the issue of accurate readout of the resistive state of devices within linear, selectorless crossbar arrays. We have presented three read-out techniques, analysed some of their key sources of errors, implemented a system capable of carrying them all out, presented measured data from a reference array and performed simulations in order to better understand the unique attributes of each technique.

Our analysis indicates that the differential read technique becomes advantageous vis-a-vis the more traditional single read if systematic offsets within the system are a significant source of error. In the noiseless, simplified simulated system we see a clear accuracy benefit although in the physical system it seems that other sources of error dominate - errors that cannot be eliminated through use of the differential read technique.

With regard to the triple read technique, we showed that it has an in-built tendency to amplify differences in the readout errors of its partial results. Interestingly, in the simulated system these 'partial errors' tended to cancel each other out, which shows that the triple read technique exhibits some degree of inherent resilience to the controlled imperfections we introduced in our simulated system. In the physical system they failed to cancel out and led to extraordinarily high RS read-out errors, possibly because of the highly randomised and asymmetric configuration of the reference array used as a test subject. Finally, we noted that the triple read technique requires employing at least two different read-out voltages throughout its cycle (in our case standard $V_{\text {read }}$ and $-V_{\text {read }}$ ). This renders it hard to operate on practical devices with asymmetric I-V curves.

Finally, we have shown simulated results indicating that none of the examined read-out techniques can truly compensate for the inherent limitations arising from within the array 
(line resistance, selectorless nature) much more successfully than the others; even when many other sources of error are factored out. This, in combination with the fact that the region of satisfactory operation in $\left(R_{l}, N\right)$-space is rather restricted points towards the absolute necessity of operating crossbar arrays with high quality selectors.

This work can be pursued further by attacking three crucial issues: a) Introducing transient analysis in order to fully investigate issues of read-out speed and power dissipation, b) measuring and simulating arrays with non-linear, possibly asymmetric IV cross-point elements and c) assessing system performance on arrays that boast selector devices, ideally 2terminal selectors integrated into the array fabric.

\section{REFERENCES}

[1] B. Govoreanu et al., " $10 \times 10 \mathrm{~nm} 2 \mathrm{hf} / \mathrm{hfo} \times$ crossbar resistive ram with excellent performance, reliability and low-energy operation," in Electron Devices Meeting (IEDM), 2011 IEEE International. IEEE, 2011, pp. 31-6.

[2] S. Pi, P. Lin, and Q. Xia, "Cross point arrays of $8 \mathrm{~nm} 8 \mathrm{~nm}$ memristive devices fabricated with nanoimprint lithography," Journal of Vacuum Science \& Technology B, vol. 31, no. 6, 2013.

[3] R. Waser and M. Aono, "Nanoionics-based resistive switching memories," Nature materials, vol. 6, no. 11, pp. 833-840, 2007.

[4] X. Yang and I.-W. Chen, "Dynamic-load-enabled ultra-low power multiple-state rram devices," Scientific reports, vol. 2, 2012.

[5] G. Indiveri et al., "Integration of nanoscale memristor synapses in neuromorphic computing architectures," Nanotechnology, vol. 24, no. 38, p. 384010, 2013.

[6] C. Kügeler et al., "High density 3d memory architecture based on the resistive switching effect," Solid-State Electronics, vol. 53, no. 12, pp. 1287-1292, 2009.

[7] H. D. Lee et al., "Integration of $4 \mathrm{f} 2$ selector-less crossbar array $2 \mathrm{mb}$ reram based on transition metal oxides for high density memory applications," in VLSI Technology (VLSIT), 2012 Symposium on. IEEE 2012, pp. 151-152.

[8] E. Linn et al., "Complementary resistive switches for passive nanocrossbar memories," Nature materials, vol. 9, no. 5, pp. 403-406, 2010.

[9] A. Chen, "A comprehensive crossbar array model with solutions for line resistance and nonlinear device characteristics," Electron Devices, IEEE Transactions on, vol. 60, no. 4, pp. 1318-1326, April 2013.

[10] G. Burr et al., "Recovery dynamics and fast (sub-50ns) read operation with access devices for $3 \mathrm{~d}$ crosspoint memory based on mixed-ionicelectronic-conduction (miec)," in VLSI Technology (VLSIT), 2013 Symposium on, June 2013, pp. T66-T67.

[11] E. Katsia et al., "Poly(3-hexylthiophene)/zno hybrid pn junctions for microelectronics applications," Applied Physics Letters, vol. 94, no. 14, pp. -, 2009. [Online]. Available: http://scitation.aip.org/content/aip/ journal/apl/94/14/10.1063/1.3114442

[12] A. Chasin et al., "High-performance a-igzo thin film diode as selector for cross-point memory application," Electron Device Letters, IEEE, vol. 35 , no. 6, pp. 642-644, June 2014.

[13] J. Y. Seok et al., "A review of three-dimensional resistive switching cross-bar array memories from the integration and materials property points of view," Advanced Functional Materials, 2014.

[14] M. Zidan et al., "Memristor multiport readout: A closed-form solution for sneak paths," Nanotechnology, IEEE Transactions on, vol. 13, no. 2, pp. 274-282, March 2014.

[15] A. Serb et al., "Limitations and precision requirements for read-out of passive, linear, selectorless rram arrays," in IEEE International Symposium on Circuits and Systems, 2015.

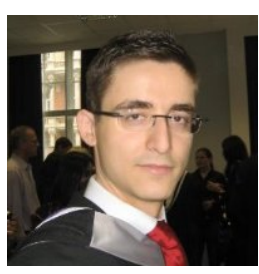

Alexander Serb Alexander Serb (M'11) received his MEng in Biomedical Engineering in 2009 and the $\mathrm{PhD}$ degree in Electrical and electronic engineering in 2013, both at Imperial College London, UK. $\mathrm{He}$ is currently a post-doctoral research fellow at the department of Electronics and Computer Science (ECS), University of Southampton, UK.

His research interests cover developing instrumentation and algorithms for testing RRAM technology, applications of resistively switching devices and neuro-inspired engineering.

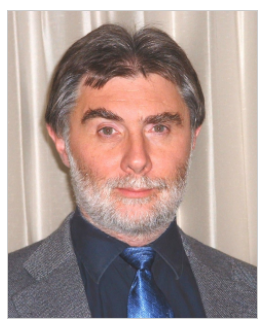

William Redman-White William Redman-White spent much of his career from 1990-2011 with NXP (and its predecessor, Philips Semiconductors) as an Engineering Fellow, specialising in analogue and RF design. Based mainly in Southampton, UK, he has also worked for Philips in San Jose, California from 2001 to 2003 and in Caen, France, in 1999. Within NXP/Philips, he worked on a wide range of projects and products, covering optical storage, cellular and WLAN radio, analogue and digital TV, and automotive applications. He was previously with Motorola, GEC-Marconi Research London, and Post Office Telecommunications UK.

Concurrent with his industrial activities, he has also held a part time academic position at Southampton University, UK, becoming full professor in 1998. His research and teaching centres around analogue and RF IC design, primarily in CMOS technology. Previous programmes included significant work on design in SOI CMOS.

He was associate editor for IEEE Journal of Solid-State Circuits from 1996 to 2002, served on ISSCC TPC from 1999-2013, and has twice been technical program chair of the European Solid State Circuits Conference. Prof. RedmanWhite is a Fellow of the IEEE and of the IET.

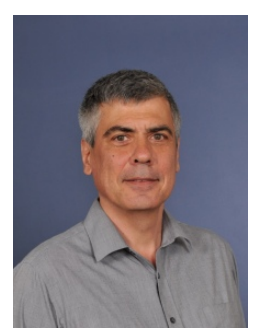

Christos Papavassiliou Christos Papavassiliou (M'96SM'05) was born in Athens, Greece, in 1960. He received the B.Sc. degree in Physics from the Massachusetts Institute of Technology, and the Ph.D. degree in Applied Physics from Yale University.

He has worked on GaAs monolithic microwave integrated circuit (MMIC) design and measurements with Foundation for Research and Technology, Hellas, Crete, Greece, and was involved in several European and regional projects on GaAs MMIC technology. Since 1996 he has been at Imperial College London, where he has worked on SiGe technology development, RF IC, and instrumentation. He has contributed to over 70 publications. His current research interests include memristor applications, electromagnetic surface wave propagation on interfaces and antenna arrays.

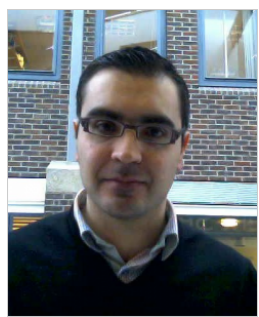

Themistoklis Prodromakis Themistoklis Prodromakis (M'08) received the B.Eng. degree in electrical and electronic engineering from Lincoln University, Lincoln, U.K., in 2003, the M.Sc. degree in microelectronic systems and telecommunications from Liverpool University, Liverpool, U.K., in 2004 and the Ph.D. degree from the Circuits and Systems Group, Imperial College London, London, U.K., in 2008. He is currently a Reader in Nanoelectronics and EPSRC Fellow affiliated with the Nano Research Group and the Southampton Nanofabrication Centre of ECS at University of Southampton. He is also a Honorary Research Fellow within Imperial College London. He previously held a Corrigan Fellowship in Nanoscale Technology and Science, funded by the Corrigan Foundation and LSI Inc., within the Centre for Bio-inspired Technology at Imperial College and a Lindemann Trust Visiting Fellowship in EECS UC Berkeley. Dr Prodromakis is a Senior Member of the IEEE, and a Member of the INE and the IET, and also serves as member of the BioCAS, Nanoelectronics and Gigascale Systems as well as the Sensory Systems Technical Committees of the IEEE Circuits \& Systems Society. His background is in Electron Devices and micro/nano-electronics processing techniques, with his research being focused on bio-inspired devices for biomedical applications. 